\title{
One-photon Absorption Characterizations, Dipole Polarizabilities and Second Hyperpolarizabilities of Chlorophyll a and Crocin
}

https://doi.org/10.1515/chem-2018-0134

received February 8, 2017; accepted August 3, 2018.

Abstract: The dispersion-free dipole polarizability $(\alpha)$ computations for chlorophyll a (1) and crocin (2) have been achieved by density functional theory (DFT) at B3LYP/ 6-31G $(d)$ level. The measured one-photon absorption (OPA) wavelengths are quite consistence with the theoretically obtained values. Ab-initio quantum chemical calculations (time-dependent Hartree-Fock (TDHF)) have been implemented to reveal the static second hyperpolarizabilities $(\gamma)$ and dynamic dipole polarizabilities of the title molecules. The examined compounds display $\gamma$ with non-zero values, introducing their third-order nonlinear optical (NLO) phenomena. The first and second frontier molecular orbitals of the investigated molecules have been also derived from DFT.

Keywords: OPA wavelength, B3LYP functional, Ab-initio computation, Third-order optical nonlinearity.

PACS: 42.65.-k, 31.15.E- .

*Corresponding author: Y. Ceylan, Selcuk University, Faculty of Sciences, Department of Physics, Campus, Konya, Turkey, E-mail: yceylan@selcuk.edu.tr

A. Karakas, M. Taser, B. B. Terlemez, N. Eren: Selcuk University, Faculty of Sciences, Department of Physics, Campus, Konya, Turkey

M. Karakaya: Department of Energy Systems, Faculty of Engineering \& Architecture, Sinop University, Sinop 57000, Turkey

Y. El Kouari, M. Lougdali: University Hassan II of Casablanca, Morocco

A. K. Arof: Centre for lonics University of Malaya, Physics Department, University of Malaya, 50603 Kuala Lumpur Malaysia B. Sahraoui: LUNAM Université, Université d'Angers, CNRS UMR 6200, Laboratoire MOLTECH-Anjou, 2 Bd Lavoisier, 49045 Angers Cedex, France

\section{Introduction}

The biological tissues are successfully imaged using linear optical (LO) and NLO procedures [1]. It has been seen in recent years that to determine the optical nonlinearity behaviour [2-10] and electro-optic applications the organic molecules with conjugate and functionalize structures have been widely investigated. The second-harmonic generation (SHG) and two-photon-excited fluorescence (TPEF) among the developed NLO procedures are extensively studied as imaging devices in the laboratories. Information about the sub-wavelength spatial arrangement for molecular nonlinear probes due to their coherent structures is provided by SHG technique rather than TPEF. The second-order optical nonlinearity only appears in noncentrosymmetric medium but is forbidden with centro-inversion symmetry. It is worth mentioning that to image the biological systems without considering any symmetry condition the third-harmonic generation (THG) is another attractive optical nonlinearity process. Rather high $\gamma$ values have been particularly obtained by carotenoids [11] and porphyrin like [12] compounds.

In this work, our aim is to present the LO and NLO behaviour of the title compounds in Figure 1 by studying dispersion-free and frequency-dependent $\alpha$ and dispersion-free $\gamma$ parameters with ab-initio and DFT calculations. Lacking the theoretical $\gamma$ determinations, the produced results here may be useful to understand the NLO phenomena of similar structures. The objective of the present study is also to characterize 1 and $\mathbf{2}$ (Figure 1) using UV-Vis spectral analysis. To find out the charge transfer effects within the title compounds, the highest occupied molecular orbitals (HOMOs) and the lowest unoccupied molecular orbitals (LUMOs) have been defined by means of DFT. 


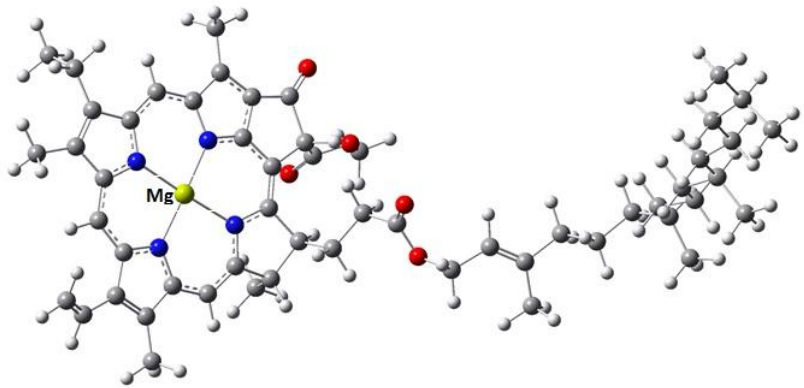

Chlorophyll a (1)

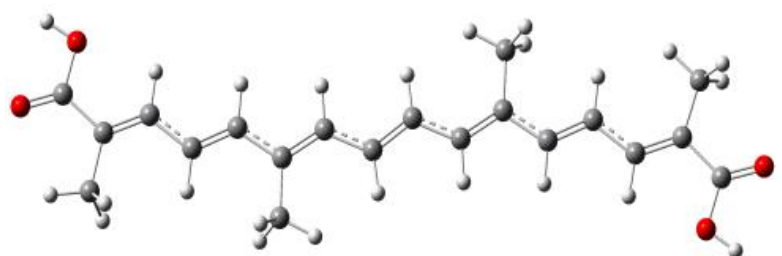

Crocin (2)

Figure 1: Chemical structures of Chlorophyll a (1) and Crocin (2).

\section{Theoretical Calculations}

We have firstly optimized the geometries of molecular structures for the title compounds. And then, the static dipole polarizabilities have been computed by the finite field (FF) approach [13]. To obtain the optimized structures and dispersion-free $\alpha$ values by DFT method at B3LYP/ 6-31G $(d)$ level, GAUSSIAN03W [14] have been utilized as package program. The orientationally averaged (isotropic) dipole polarizabilities $\langle\alpha\rangle$ are expressed as follows [15]:

$\langle\alpha\rangle=\left(\alpha_{\mathrm{x}}+\alpha_{\mathrm{y}}+\alpha_{\mathrm{z}}\right) / 3$

The dynamic hyperpolarizability values are often carried out using self-consistent field (SCF) technique (so-called TDHF) [16]. The TDHF procedure of the GAMESS [17] package program performed $\gamma(0 ; 0,0,0)$ at $\omega=0$ and $\alpha(-\omega ; \omega)$ calculations at $\omega=0.04282$ atomic units (a.u.) $(\lambda=1064 \mathrm{~nm})$ with $6-31 \mathrm{G}(d)$ polarized basis set. The dispersion-free second hyperpolarizability is defined as $\gamma(0 ; 0,0,0)$. The computations using 6-31G $(d)$ polarized basis set is to find out quite certain NLO parameters. Accurate hyperpolarizability calculations need to truly choice of basis sets. In general, since the molecular hyperpolarizabilities also need to know the explanation of the wave function tails, the diffuse and/ or polarization functions should be included in the basis sets for supporting the convergence of the property [18]. Bartlett et al. [19] show that the dispersion-free (hyper) polarizabilities require to use large basis sets. Kenawi et al. [20] performed the (hyper)polarizability computations with various polarized and / or diffused basis sets at DFT and Hartree-Fock levels of theory for diclofenac sodium. The best (hyper)polarizability values for the studied diclofenac sodium have been obtained from the calculations with polarized and diffuse functions [20]. The (hyper)polarizabilities are computed by the numerical derivatives of the electric dipole moments according to the applied electric fields, so their computed values might be affected by the basis set properties. The various basis sets with polarized and diffused functions could be utilized for computing the (hyper)polarizabilities. Maroulis [18] reported that the types of valence polarization need to truly choice of basis sets. He mentioned that because of the large size of trans-butadiene, the involvement of electron correlations needs a strict study of the basis set effects [18]. It is expected that $6-31 \mathrm{G}(d)$ polarized basis set utilized in this work seems to find out the molecular properties with near Hartree-Fock accuracy.

The averaged (isotropic) second hyperpolarizabilities $\langle\gamma\rangle$ have been computed as follows:

$\langle\gamma\rangle=(1 / 5)\left[\gamma_{x x x x}+\gamma_{y y y y}+\gamma_{z z z z}+2\left(\gamma_{x x y y}+\gamma_{x x z z}+\gamma_{y y z z}\right)\right]$

In this work, to determine the OPA wavelengths $\left(\lambda_{\max }\right)$ of 1-2, we utilized the CIDRT group which indicates the configuration interaction (CI) with all doubly occupied molecular orbitals from Hartree-Fock reference determinant. The $\lambda_{\text {max }}$ calculations have been directly performed by means of TDHF procedure using 6-31G basis set implemented in the GAMESS [17] program. The calculations on OPA wavelengths do not need to have any polarized basis sets including $d$ polarized functions. So, we used 6-31G basis set without adding a $d$ polarized function to obtain $\lambda_{\max }$ values of the lowest lying electronic transitions for the studied compounds. All frequency dependent dipole polarizabilities, hyperpolarizabilities and transition 


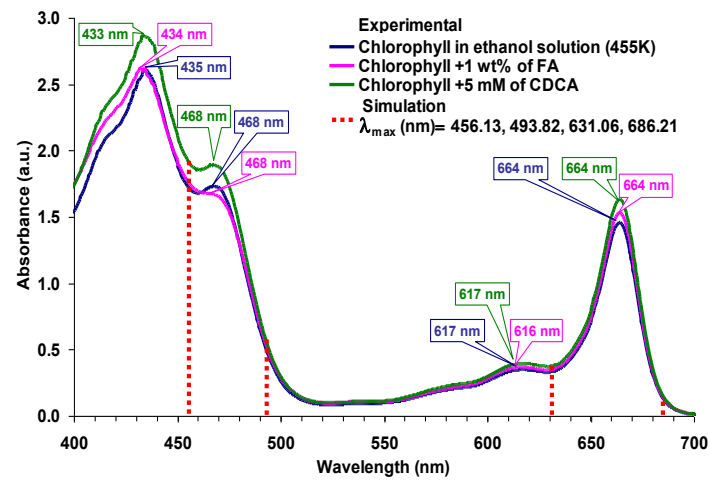

Chlorophyll a (1)

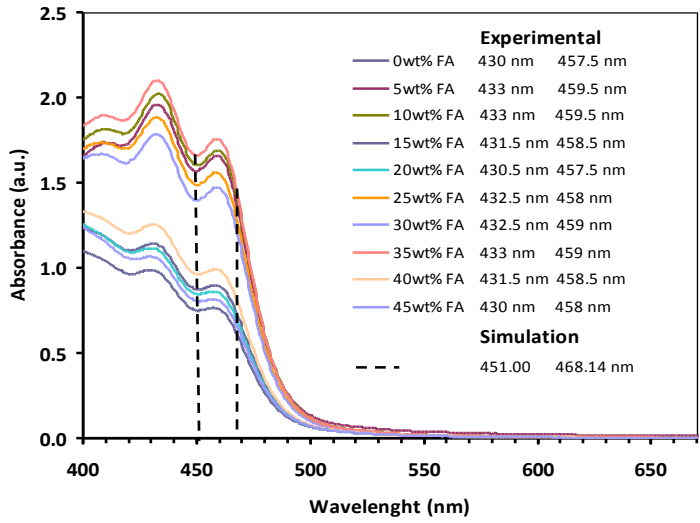

Crocin (2)

Figure 2: Experimental UV-Vis spectra and theoretical simulations of Chlorophyll a (1) and Crocin (2).

wavelengths have been generated on a PC with an Intel (R) core (TM) I7-2630QM operator, 5.8 GB RAM memory and 2 $\mathrm{GHz}$ frequency using Linux PC GAMESS version running under Linux Fedora release 11 (Leonidas) environment.

To explore the relationship between optical nonlinearity and molecular structure; the first and second frontier molecular orbitals and HOMO-LUMO energy band gaps have been evaluated by GAUSSIANO3W [14] program using DFT/ B3LYP with 6-31G $(d)$ basis set. The HOMO-LUMO energy gaps $\left(\mathrm{E}_{\mathrm{g}}\right)$ are calculated using the following equation:

$$
\mathrm{E}_{\mathrm{g}}=\mathrm{E}_{\text {LUMO }}-\mathrm{E}_{\text {HOMO }}
$$

\section{Computational Results and Discussion}

In this work, the maximum OPA wavelengths for the title molecules have been examined within $400-700 \mathrm{~nm}$ by UVVis recording spectrometer. The UV-Vis patterns of $\mathbf{1}$ have been taken from chlorophyll a, solved in ethanol, $1 \mathrm{wt} \% 1$ of ferulic acid (FA) and also $5 \mathrm{mM}$ of chenodeoxycholic acid (CDCA) as shown in Figure 2. The UV-Vis patterns of 2 have been taken from 0-45 wt \% solution of FA as shown in Figure 2. The main features of the absorption spectra of 1 consists of two allowed transitions in the blue spectral region referred to as the Soret band (B band) and two weaker transitions in red or near IR region referred to as the $\mathrm{Q}$ band. These bands arise from $\pi$ to $\pi^{*}$ transitions of four frontier orbitals. The absorption spectrum of 1 has been characterized by two Soret bands at about 433 $\mathrm{nm}$ (stronger one) and at $468 \mathrm{~nm}$ (relatively weak one), and also a strong $\mathrm{Q}$ band near $664 \mathrm{~nm}$ with a weak Q band near $616 \mathrm{~nm}$ (see Figure 2). It has been found that 2 has two absorption bands at around $430 \mathrm{~nm}$ and 457 $\mathrm{nm}$ in the visible region as shown in Figure 2. The UVVis absorption spectra have been obtained, showing the vertical transition energies from ground level to excited level. Figure 2 also shows the simulation values for OPA wavelengths. Theoretically obtained $\lambda_{\max }$ values for $\mathbf{1}$ and 2 are reasonably in accordance with the experimental ones. It is seen from Figure 2 that the computed $\lambda_{\text {max }}$ values are systematically a bit higher than the measurement results. The higher estimated energies are related to the reduced CI technique, correlating the ground levels but not excited levels. The multi-reference configuration interaction (MRCI) procedure includes the excitations inferred from both ground level electronic configuration and also a few excited levels. The Slater determinants obtained by the excitations are known as reference Slater determinants. To use more than one reference configuration results in better correlations and much decreased energy values. Therefore, using MRCI computation, one can have more powerful correlation effects between the ground and excited levels of the molecular structures. The quantitatively correct excitation energies might be attained by carefully selection of the references. To take into account the highest configuration of an excited level in the reference space generates much decreased energy value for the excited configuration. As a result, much higher excitation energies produced by CI method are reduced. MRCI might supply important improvement on the computed $\lambda_{\max }$ values of the examined compounds. But, MRCI is rather time consuming and so expensive procedure to get such values computationally. 
Table 1: Some selected components of the static $\alpha(0 ; 0)$ and $\langle\alpha\rangle(0 ; 0)\left(\times 10^{-24}\right.$ esu $)$ values for 1-2.

\begin{tabular}{llccc}
\hline Compound & $\alpha_{x x}$ & $\alpha_{y y}$ & $\alpha_{z z}$ & $\langle\alpha\rangle$ \\
\hline $\mathbf{1}$ & 110.805 & 111.129 & 125.148 & 115.694 \\
$\mathbf{2}$ & 183.361 & 31.866 & 14.821 & 76.682 \\
\hline
\end{tabular}

Table 2: All static $\gamma(0 ; 0,0,0)$ components and $\langle\gamma\rangle(0 ; 0,0,0)\left(\times 10^{-37}\right.$ esu) values for $\mathbf{1 - 2}$

\begin{tabular}{rrrrrrrr}
\hline & $\gamma_{x x x x}$ & $\gamma_{y y y y}$ & $\gamma_{z z z z}$ & $\gamma_{x x y y}$ & $\gamma_{x x z z}$ & $\gamma_{y y z z}$ & $\langle\gamma\rangle$ \\
\hline $\mathbf{1}$ & 94.980 & 111.296 & 1973.129 & 65.876 & -15.052 & -26.765 & 445.504 \\
$\mathbf{2}$ & 26838.688 & -4.998 & -0.683 & -167.524 & 1.310 & 1.747 & 5300.814 \\
\hline
\end{tabular}

Table 3: Some selected components of the frequency-dependent $\alpha(-\omega ; \omega)$ and absolute values of $\langle\alpha\rangle(-\omega ; \omega)\left(\times 10^{-24}\right.$ esu $)$ at $\omega=0.04282$ a.u. for 1-2.

\begin{tabular}{lllll}
\hline Compound & $\alpha_{x x}$ & $\alpha_{y y}$ & $\alpha_{z z}$ & $\langle\alpha\rangle$ \\
1 & 66.431 & 71.722 & 86.925 & 75.026 \\
$\mathbf{2}$ & 117.989 & 21.108 & 6.076 & 48.391 \\
\hline
\end{tabular}

Tables 1-2, respectively, list a few important computed components for dispersion-free dipole polarizabilities and second hyperpolarizabilities of 1-2. The static $\langle\alpha\rangle$ values in Table 1 have an apparent reduction in sort order $\mathbf{1}>\mathbf{2}$, while the static $\langle\gamma\rangle$ values show a reduction in sort order $\mathbf{2}>\mathbf{1}$ in Table 2. Compound $\mathbf{1}$ has lower static cubic $\langle\gamma\rangle$, and thereby it is seen that compound $\mathbf{2}$ has much better third-order NLO phenomena than compound $\mathbf{1}$.

A few important computed components for dynamic dipole polarizabilities of $\mathbf{1 - 2}$ are shown in Table 3. The dynamic $\langle\alpha\rangle$ values and their corresponding static ones display exactly same reduction in sort order $\mathbf{1}>\mathbf{2}$ (see Tables 1,3).

The calculated first and second frontier molecular orbital energies and also energy band gaps for 1-2 are presented in Table 4. Figure 3 shows the first and second frontier MOs. It has been found that the first and second frontier molecular orbital energy band gaps give a reduction in sort order $\mathbf{2} \geq \mathbf{1}$. There is a certain relationship between hyperpolarizabilities and HOMO-LUMO band gaps. The HOMO-LUMO energy band gaps with lower values can achieve better hyperpolarizabilities. B (Soret) and $Q$ transitions can be easily observed by UV-Vis spectral analysis of 1 in Figure 2 [21]. The transitions among HOMO / HOMO-1 and LUMO / LUMO+1 could be represented by $\mathrm{Q}$ and $\mathrm{B}$ bands, respectively. The frontier orbitals for $\mathbf{1}$ are placed on the porphyrin chain, whereas there is no centering on the phytol chain. As a result, many electronic transitions related to $\mathbf{1}$ will originate from the porphyrin chain. A major contribution of structural symmetry for porphyrin chain still existing in $\mathbf{1}$ even with the existence of some functional substituents on the domain of the chain is introduced into quite uniform spatial distribution of orbital among the pairs of first and second frontier. The electronic transition of molecule containing the photons which provide high oscillator strengths for upper and lower transitions is mainly supported by uniform spatial range between occupied and unoccupied frontier molecular orbitals. The first frontier highest occupied and lowest unoccupied molecular orbitals for $\mathbf{2}$ display almost the same charge-density transmission (see Table 4).

\section{Conclusions}

Attempts have been made in the present work for the OPA characterizations and second hyperpolarizability responses of 1-2 from the UV-Vis spectra and quantum chemical calculations. The assignments made to obtain the OPA wavelengths of $\mathbf{1}$ and $\mathbf{2}$ by means of CI method with coherent variations from the measured data seem to be correct. The dipole polarizability and third-order 


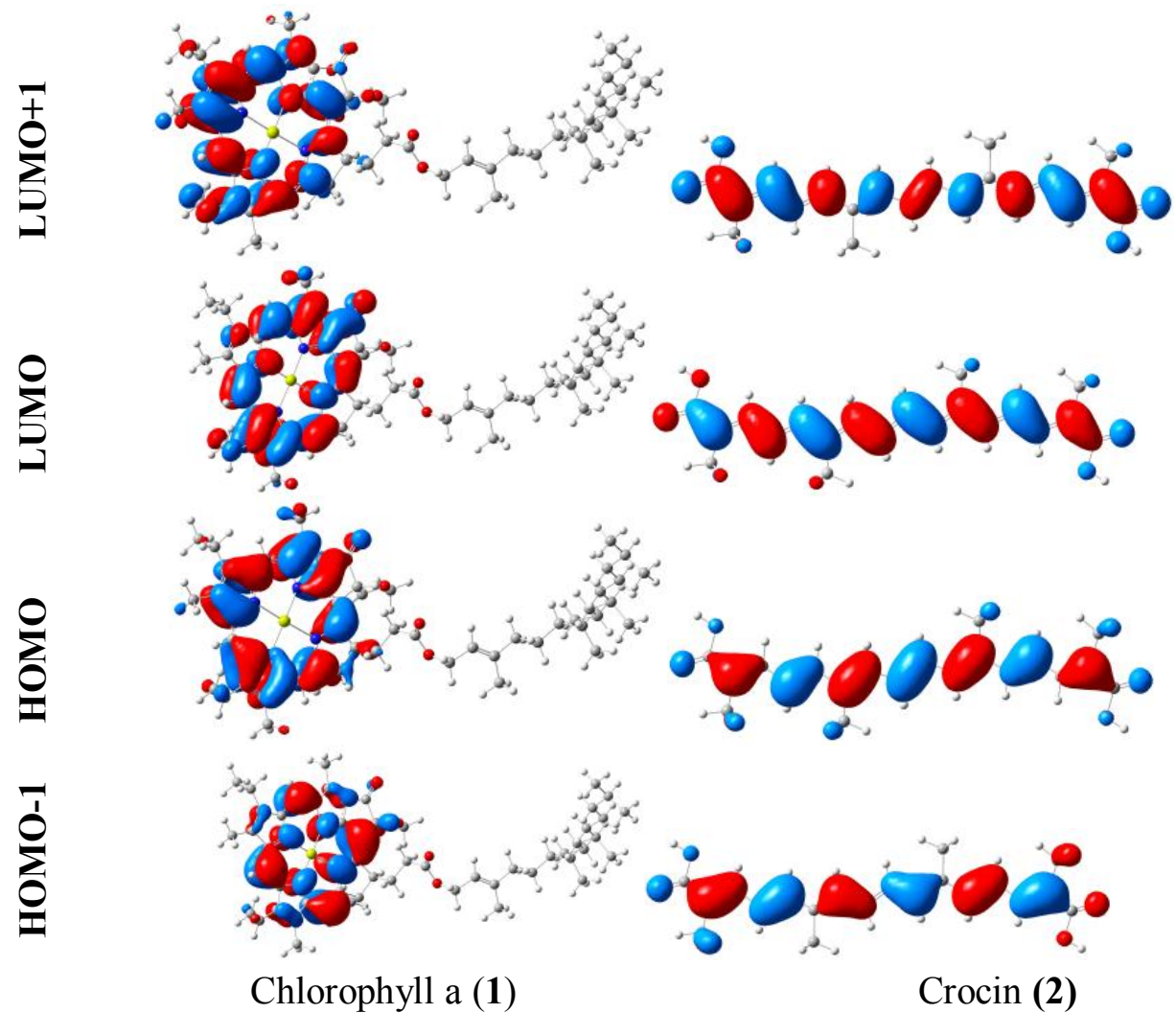

Figure 3: The frontier and second frontier molecular orbitals of Chlorophyll a (1) and Crocin (2).

Table 4: Calculated HOMO-LUMO energy (a.u.) and HOMO-LUMO band gap $\left(E_{g}\right)$ values for 1-2.

\begin{tabular}{lll}
\hline & $\mathbf{1}$ & $\mathbf{2}$ \\
\hline HOMO & -0.18035 & -0.19032 \\
LUMO & -0.09235 & -0.10037 \\
E $_{\mathrm{g}}$ HOMO-LUMO $]$ & 0.08800 & 0.08995 \\
HOMO-1 & -0.19152 & -0.22949 \\
LUMO +1 & -0.06404 & -0.06031 \\
E $_{\mathrm{g}}$ (HOMO-1)-(LUMO+1)] & 0.12748 & 0.16918 \\
\hline
\end{tabular}

hyperpolarizability values of $\mathbf{1 - 2}$ have been computed using DFT/ B3LYP (for static $\alpha$ ) and TDHF (for dynamic $\alpha$ and static $\gamma$ ) procedures. The calculated second hyperpolarizabilities of the title compounds are non-zero, verifying microscopic third-order optical nonlinearity behaviour. The HOMO and LUMO analyses have been also used to find out the charge transfers within the examined structures.

\section{References}

[1] Denk W., Piston D.W., Webb W.W., Handbook of biological confocal microscopy, Pawley J. (Ed.), Springer, New York, 2006.

[2] Sahraoui B., Nguyen Phu X., Sallé M., and Gorgues A., Electronic and nuclear contributions to the third-order nonlinear optical susceptibilities of new $\mathbf{p}-\mathbf{N}, \mathbf{N}$-dimethylaniline tetrathiafulvalene derivatives, Optics Letters, 1998, 23 (23), 1811-1813, DOI:10.1364/OL.23.001811.

[3] Derkowska B., Mulatier J.C., Fuks I., Sahraoui B., Nguyen Phu X., Andraud C., Third-order optical nonlinearities in new octupolar molecules and their dipolar subunits, J. Opt. Soc. Am. B, 2001, 18 (5), 610-616. DOI: 10.1364/JOSAB.18.000610.

[4] Apostoluk A., Chapron D., Gadret G., Sahraoui B., Nunzi J-M., Fiorini-Debuisschert C., et al., Quasi-phase-matched gratings printed by all-optical poling in polymer films, Optics Letters, 2002, 27(22), 2028-2030. DOI: 10.1364/OL.27.002028.

[5] Kolev Ts., Kityk I.V., Ebothe J., Sahraoui B., Intrinsic hyperpolarizability of 3-dicyanomethylene-5,5-dimethyl-1[2-(4-hydroxyphenyl)ethenyl]-cyclohexene nanocrystallites incorporated into the photopolymer matrices, Chemical Physics Letters, 2007, 443, 309-312. DOI: 10.1016/j.cplett.2007.06.051.

[6] Papagiannouli I., Iliopoulos K., Gindre D., Sahraoui B., Krupka O., Smokal V., et al., Third-order nonlinear optical response of push-pull azobenzene polymers, Chemical Physics Letters, 2012, 554, 107-112. DOI: 10.1016/j.cplett.2012.10.007. 
[7] Sahraoui B., Luc J., Meghea A., Czaplicki R., Fillaut J-L. and Migalska-Zalas A., Nonlinear optics and surface relief gratings in alkynyl-ruthenium complexes, J. Opt. A: Pure Appl. Opt., 2009, 11, 024005, pp. 26. DOI: 10.1088/14644258/11/2/024005.

[8] Sahraoui B., Sylla M., Bourdin J.P., Rivoire G., Zaremba J., Nguyen T.T., et al., Third-order Nonlinear Optical Properties of Ethylenic Tetrathiafulvalene Derivatives, Journal of Modern Optics, 1995, 42(10), 2095-2107. DOI: 10.1080/09500349514551821.

[9] Terkia-Derdra N., Andreu R., Salle M., Levillain E., Orduna J., Garin J., et al., $\pi$ conjugation across the tetrathiafulvalene core: synthesis of extended tetrathiafulvalene derivatives and theoretical analysis of their unusual electrochemical properties, Chem. Eur. J., 2000, 6(7), 1199-1213.

[10] Derkowska B., Wojdyła M., Czaplicki R., Bała W., Sahraoui B., Influence of the central metal atom on the nonlinear optical properties of MPcs solutions and thin films, Optics Communications, 2007, 274(1), 206-212. DOI: https://doi. org/10.1016/j.optcom.2007.01.067.

[11] Hermann J.P., Ducuing J., Third-order polarizabilities of longchain molecules, J. Appl. Phys., 1974, 45 (11), 5100-5102. DOI: 10.1063/1.1663197.

[12] Senge M.O., Fazekas M., Notaras E.G.A., Blau W.J., Zawadzka M., Locos O.B., et al., Nonlinear optical properties of porphyrins, Adv. Mater., 2007, 19, 2737-2774. DOI: 10.1002/ adma.200601850.

[13] Kurtz H.A., Stewart J.J.P., Dieter K.M., Calculation of the nonlinear optical properties of molecules, J. Comput. Chem., 1990, 11(1), 82-87. DOI: 10.1002/jcc.540110110.

[14] Frisch M.J. et. al., Gaussian 03, Revision E.01 (Gaussian, Inc., Wallingford CT, 2004).

[15] Bogaard M.P., Orr B.J., MTP international review of science, Buckingham A.D. (Ed.), Butterworths, London, 1975, Vol. 2, p. 149.

[16] Shelton D.P., Rice J.E., Measurements and calculations of the hyperpolarizabilities of atoms and small molecules in the gas phase, Chem. Rev., 1994, 94(1), 3-29. DOI: 10.1021/ cr00025a001.

[17] Intel $\times 86$ (win32, Linux, OS/2, DOS) version. PC GAMESS version 6.2, build number 2068. This version of GAMESS is described in: Schmidt M.W. et. al., General atomic and molecular electronic structure system, J. Comput. Chem., 1993, 14 (11), 1347-1363, DOI: 10.1002/jcc.540141112.

[18] Maroulis G., On the accurate theoretical determination of the static hyperpolarizability of trans-butadiene, J. Chem. Phys., 1999, 111(2), 583-591. DOI : 10.1063/1.479339.

[19] Bartlett R.J., Sekino H., Purvis G.D., Comparison of MBPT and coupled-cluster methods with full $\mathrm{Cl}$. Importance of triplet excitation and infinite summations, Chem. Phys. Lett., 1983, 98(1), 66-71. DOI: 10.1016/0009-2614(83)80204-8.

[20] Kenawi I.M., Kamel A.H., Hilal R., BSSE effects on the static dipole polarizability and first dipole hyperpolarizability of diclofenac sodium, J. Mol. Struct. (Theochem), 2008, 851(1-3), 46-53. DOI: 10.1016/j.theochem.2007.10.034.
[21] Linnanto J., Korppi-Tommola J., Spectroscopic properties of Mg-chlorin, Mg-porphin and chlorophylls $a, b, c_{1}, c_{2}, c_{3}$ and $d$ studied by semi-empirical and $a b$ initio $\mathrm{MO} / \mathrm{Cl}$ methods, Phys. Chem. Chem. Phys., 2000, 2, 4962-4970. DOI: 10.1039/b004998k. 